\title{
PARAMETRIZATIONS OF POSITIVE MATRICES WITH APPLICATIONS
}

\author{
M. C. TSENG, H. ZHOU, AND V. RAMAKRISHNA
}

\begin{abstract}
The purpose of this work is twofold. The first is to survey some parametrizations of positive matrices which have found applications in quantum information theory. The second is to provide some more applications of a parametrization of quantum states and channels introduced by T. Constantinescu and the last author, and thereby to provide further evidence of the utility of this parametrization. This work is dedicated to the memory of our colleague and teacher, the late Professor T. Constantinescu.
\end{abstract}

\section{INTRODUCTION}

Positive matrices play a vital role in quantum mechanics and its applications (in particular, quantum information processing). Indeed the two basic ingredients in the theory of quantum information, viz., quantum states and quantum channels involve positive matrices. See, for instance, [17, 21]. Thus, a study of parametrizations of positive matrices seems very much warranted. In particular, the very useful Bloch sphere picture, 17, 21], for the quantum state of a qubit has prompted several attempts at the extension of this picture to higher dimensions. In the process, several groups of researchers have looked into the question of finding tractable characterizations of positive matrices, which could lead to useful parametrizations of positive matrices, 3, 15, 23, 27, 5.

This paper is organized as follows. In the next section we set up basic notation and also point out some sources for positive matrices in quantum mechanics and its applications. The third section introduces six (perhaps well-known) characterizations of positive matrices, and reviews some putative parametrizations of states of qudits. In the next section, we review a parametrization proposed in [5], reiterating its utility. The final section offers two more applications of the parametrization in [5]. The first concerns Toeplitz states, i.e., density matrices which are also Toeplitz. The second investigates constraints imposed on relaxation rates of an open quantum system by the requirement of complete positivity.

\section{Sources of Positive Matrices in Quantum Theory}

Let us recall that a matrix is positive semidefinite (positive, for short) if $z^{*} P z \geq 0$ for all $z \in C^{n}$. One can easily extend this definition to infinite positive matrices. In effect such a matrix is what is called a positive kernel, [6, viz., a map $K: N_{0} \times N_{0} \rightarrow C$, 
where $N_{0}$ is the set of non-negative integers, with the property that for each $n>0$, and each choice $p_{1}, \ldots, p_{n}$ in $N_{0}$ and each choice $z_{1}, \ldots, z_{n}$ of elements of $C$ we have

$$
\sum_{i, j=1}^{n} K\left(p_{i}, p_{j}\right) \bar{z}_{i} z_{j} \geq 0
$$

Positive matrices intervene in at least two of the basic ingredients of quantum mechanics and quantum information theory viz., quantum states and quantum channels. There are, of course, more sources for positive matrices, but, for reasons of brevity, we will confine ourselves to discussing states and channels.

The state of a $d$-dimensional quantum system is described by a $d \times d$ positive density matrix of trace 1 , that is, a positive element of trace 1 in the algebra $\mathcal{M}_{d}$ of complex $d \times d$ matrices. States described by rank one density matrices are called pure states.

A quantum channel is a completely positive map $\Phi: \mathcal{A} \rightarrow \mathcal{L}(\mathcal{H})$ from a $C^{*}$-algebra $\mathcal{A}$ into the set $\mathcal{L}(\mathcal{H})$ of all bounded linear operators on the Hilbert space $\mathcal{H}$ (in the situations most frequently met in quantum information processing, $\mathcal{A}=\mathcal{M}_{d}$ and $\left.\mathcal{L}(\mathcal{H})=\mathcal{M}_{d^{\prime}}\right)$. By the Stinespring theorem, [19, Theorem 4.1, such a map is the compression of a $*$-homomorphism. For $\mathcal{A}=\mathcal{M}_{d}$, there is a somewhat more explicit representation, given in [4] (see also [13]). Thus, $\Phi: \mathcal{M}_{d} \rightarrow \mathcal{L}(\mathcal{H})$ is completely positive if and only if the matrix

$$
S=S_{\Phi}=\left[\Phi\left(E_{k, j}\right)\right]_{k, j=1}^{d}
$$

is positive, where $E_{k, j}, k, j=1, \ldots, d$, are the standard matrix units of $\mathcal{M}_{d}$. Each $E_{k, j}$ is a $d \times d$ matrix consisting of 1 in the $(k, j)$ th entry and zeros elsewhere.

Remark 1. Usually one requires a quantum channel to satisfy two additional requirements: i) $\Phi$ be trace preserving, and/or ii) $\Phi$ be unital.

A Kraus operator representation of a completely positive map is a (non-unique) choice of operators $V_{i}$ such that one can express the effect of $\Phi$ via

$$
\Phi(\rho)=\sum_{i=1}^{r} V_{i} \rho V_{i}^{*}
$$

Usually only the non-zero $V_{i}$ are taken into account in the above equation (though sometimes it is convenient to ignore this convention).

Then $\Phi$ is trace-preserving iff $\sum_{i=1}^{r} V_{i}^{*} V_{i}=\mathrm{I} d$, while $\Phi$ is unital iff $\sum_{i=1}^{r} V_{i} V_{i}^{*}=\mathrm{I} d$. These properties can also be verified (without any reference to Kraus representations) by computing the partial traces of $S_{\Phi}$ viewed as an unnormalized state (see [26]).

All choices of Kraus operator representations for $\Phi$ come from square-roots of $S_{\Phi}$, i.e., matrices $T$ such that $S_{\Phi}=T T^{*}$. One then obtains the $V_{i}$ from the $i$ th column of $T$ by reversing the vec operation, [10, 26]. Recall that the vec operator associates to a $d \times e$ matrix, $V$, a vector in $C^{d e}$ obtained by stacking the columns of $V$. It is precisely because of lack of uniqueness in the square roots of $S_{\Phi}$ that the Kraus operator representation of $\Phi$ is non-unique. 
We should point out that some of the definitions for quantum channel notions used in [5], though equivalent to the standard ones (i.e., the ones used here), are different.

\section{Characterizations of Positive Matrices}

All positive matrices are Hermitian (unlike the real case, the definition of a positive matrix automatically forces Hermiticity). There are several characterizations of positive matrices as a subclass of Hermitian matrices. Some of these yield useful parametrizations of positive matrices.

The following theorem, which for the most part is standard textbook material (see, for instance, the classic [11]), reviews some of these characterizations.

Theorem 2. Let $P$ be a Hermitian matrix. Then the following are equivalent:

- P1 P is positive.

- P2 All the eigenvalues of $P$ are non-negative.

- P3 There is an upper-triangular matrix $T$ such that $P=T^{*} T$ (Cholesky decomposition)

- P4 All principal minors of $P$ are non-negative.

- P5 Let $p(t)=t^{n}+\sum_{i=0}^{n-1}(-1)^{i} b_{i} t^{n-i}$ be the characteristic polynomial of $P$. Then $b_{i} \geq 0$, for all $i$.

- P6 There is a Hermitian matrix $H$ such that $P=H^{2}$.

Remark 3. i) P3 is normally mentioned only for positive definite matrices in the bulk of the literature. However, a limiting argument shows that it is valid for positive semidefinite matrices as well. ii) That $\mathbf{P 5}$ is equivalent to P2 is just a consequence of Descartes' rule of signs. iii) $\mathbf{P} 4$ should be folklore. Quite surprisingly, we were unable to find any source where $\mathbf{P} \mathbf{4}$ is stated explicitly (even in a venerable text such as [11]). Since a similar statement for positive definite matrices (viz., positive definiteness is equivalent to the positivity of the leading principal minors) is well documented and we have seen this statement occassionally incorrectly applied to positive semidefinite matrices, we will include a brief proof here. Clearly if $P$ is positive, all principal submatrices of $P$ are positive, and hence all principal minors are non-negative. Conversely suppose all principal minors of $P$ are non-negative. Since the coefficients $b_{i}$ of the characteristic polynomial of any matrix are just the sum of all the $i \times i$ principal minors of $P$, it follows that $b_{i} \geq 0$. Hence $P$ is positive.

Whilst the above conditions are equivalent to positivity, they typically do not lead to useful parametrizations of positive matrices. For instance, parametrizing $P$ by its eigenvalues only describes the $U(n)$ orbit to which $P$ belongs. For the same reason one cannot parametrize $P$ by the coefficients $b_{i}$ of the characteristic polynomial $p(t)$.

However, one can turn these characterizations into potential parametrizations. To illustrate this consider the problem of parametrizing quantum states in dimension $d$, i.e., $d \times d$ positive matrices with unit trace. The standard starting point is to represent 
a state $\rho$ via

$$
\rho=\frac{1}{d}\left(I_{d}+\sum_{i=1}^{d^{2}-1} \beta_{i} \lambda_{i}\right)
$$

Here $\beta_{i} \in R$ and the $\lambda_{i}$ form an orthogonal basis for the space of traceless Hermitian matrices, specifically $\operatorname{Tr}\left(\lambda_{i} \lambda_{j}\right)=2 \delta_{i j}$. One typical choice is the so-called generalized Gell-Mann matrices, [15, 27]. This basis is obtained from the matrices $E_{k j}, k, j=$ $1, \ldots, d\left(E_{k j}=e_{k} e_{j}^{*}\right)$ via the following construction:

$$
\begin{gathered}
f_{k, j}^{d}=E_{k, j}+E_{j, k}, \quad k<j, \\
f_{k, j}^{d}=\frac{1}{i}\left(E_{j, k}-E_{k, j}\right), \quad k>j, \\
h_{1}^{d}=I_{d}, \quad h_{k}^{d}=h_{k}^{d-1} \oplus 0, \quad 1<k<d, \quad h_{d}^{d}=\sqrt{\frac{2}{d(d-1)}}\left(h_{1}^{d-1} \oplus(1-d)\right) .
\end{gathered}
$$

These matrices, $f_{k, j}^{d}, h_{1}^{d}, h_{k}^{d}, h_{d}^{d}$ together form one choice of the $\left\{\lambda_{i}, I_{d}\right\}$ basis for the space of $d \times d$ Hermitian matrices. When $d=2$ this is precisely the Pauli matrix basis. When $d=3$ one gets the usual Gell-Mann matrices.

With Equation (3.1) as the starting point one can restrict the vector $\beta=\left(\beta_{1}, \ldots, b_{d^{2}-1}\right) \in$ $R^{d^{2}-1}$ to satisfy any of the characterizations $\mathbf{P 1}-\mathbf{P} 6$. In principle, this provides a bijection from a subset of $R^{d^{2}-1}$, say $D_{\beta}$, to the space of $d \times d$ density matrices. This is precisely what is proposed simultaneously in [15, 3] for the characterization P5. However, now by conservation of difficulty, the burden of the analysis of quantum states in dimension $d>2$ is shifted to obtaining a concrete analysis of the subset $D_{\beta}$. In particular, these do not lead to easily computed parametrization of quantum states (cf., the conclusions section of [15]). Interestingly enough each of these characterizations leads precisely to the Bloch sphere picture when $d=2$, as we encourage the reader to verify. However, this approach has some utility in higher dimensions as well. For instance, depending on which characterization one uses, it is at least possible to be more precise about the set of pure states (i.e, rank one states). We shall explain this via the characterization $\mathbf{P} 6$ because pure states are precisely those states, $\rho$ for which $\rho^{2}=\rho$, and this fits in nicely with $\mathbf{P 6}$.

In order to state a precise result, let us introduce the tensor $d_{k l i}$ obtained from considering the Jordan structure of the $\lambda_{i}$. Specifically, if $\left\{\lambda_{k}, \lambda_{l}\right\}$ denotes the Jordan commutator of $\lambda_{k}, \lambda_{l}$, then

$$
\left\{\lambda_{k}, \lambda_{l}\right\}=\lambda_{k} \lambda_{l}+\lambda_{l} \lambda_{k}=\frac{4}{d} I_{d} \delta_{k l}+\sum_{i=1}^{d^{2}-1} d_{k l i} \lambda_{i}
$$

We use the $d_{k l i}$ to introduce an operation amongst vectors $x, y \in R^{d^{2}-1}$, via

$$
x \cup y=\left(\sum_{j, k=1}^{d^{2}-1} d_{1 j k} x_{j} y_{k}, \sum_{j, k=1}^{d^{2}-1} d_{2 j k} x_{j} y_{k}, \ldots, \sum_{j, k=1}^{n^{2}-1} d_{i j k} x_{j} y_{k}, \ldots\right)
$$


$x \cup y$ is thus a vector in $R^{d^{2}-1}$. We can now state

Proposition 4. Every density matrix can be represented in the form in Equation (3.1) with $\beta=\frac{2 \kappa}{d} \beta_{0}+\frac{\beta_{0} \cup \beta_{0}}{d}$, where $\beta_{0}$ is any vector in $R^{d^{2}-1}$ with $\left\|\beta_{0}\right\|^{2} \leq \frac{d^{2}}{2}$ and $\kappa=+\sqrt{\frac{d^{2}-2\left\|\beta_{0}\right\|^{2}}{d}}$. Conversely any Hermitian matrix admitting such a representation is necessarily a density matrix. $\rho$ is pure precisely if it can be represented in the form in Equation 3.1) with $<\beta, \beta>=\frac{d^{2}-d}{2}$ and $(d-2) \beta=\beta \cup \beta$.

The proof is straightforward. Since $\rho=H^{2}$ and $H$ itself can be expanded as a linear combination of $I_{d}$ and the $\lambda_{i}$ (albeit with the coefficient of $I_{d}$ different from $\frac{1}{d}$ ), the first part of the result follows from the linear independence of $\left\{I_{d}, \lambda_{i}\right\}$. For the second part, we represent $\rho$ as in Equation (3.1) and equate it to its square.

Once again, the difficulty is in the analysis of states which are not pure. It is worth mentioning that the pure state condition in the above proposition is essentially the same as that obtained from the characterization P5 (for a pure state the characteristic polynomial is $p(t)=t^{d}+(-1)^{d} t^{d-1}$, i.e., $\left.b_{1}=1, b_{i}=0, i \geq 2\right)$.

It should be pointed out that even an analysis of the pure state conditions is far from trivial. The condition $(d-2) \beta=\beta \cup \beta$ is vacuously true when $d=2$ (since the Pauli matrices anti-commute). For $d \geq 3$, this condition imposes genuine restrictions. It is an interesting problem to find an orthogonal basis for the space of Hermitian matrices (the generalized Gell-Mann matrices form just one amongst many) which is close to "abelian", i.e, one for which many of the $d_{k l i}$ vanish, to facilitate the analysis of the condition $(d-2) \beta=\beta \cup \beta$.

In contrast, the parametrization discussed in the next section yields a very simple characterization of pure states.

\section{A DifFEREnt PARAmetrization of Positive MATRICES}

In this section we recall informally the main result of [5] on the parametrization of positive matrices. In order to do that a few preliminary definitions and notions are needed.

To any contraction $T$, one defines its defect operator via

$$
D_{T}=\left(I-T^{*} T\right)^{1 / 2}
$$

Here $M^{*}$ is the adjoint of an operator (when $M$ is a scalar, this is merely complex conjugation).

To such a contraction one can also associate a certain unitary operator, called the Julia operator of $T$ via

$$
U(T)=\left[\begin{array}{cc}
T & D_{T^{*}} \\
D_{T} & -T^{*}
\end{array}\right]
$$

Thus, $U(T)$ is a unitary dilation of $T$. 
If we are given a family of contractions $\Gamma_{k j}, j \geq k$ with $\Gamma_{k k}=0$ for all $k$, then we associate to it a family of unitary operations via the Julia operator construction as follows. We first let $U_{k, k}=\mathrm{I} d$, while for $j>k$ we set

$$
U_{k, j}=U_{j-k}\left(\Gamma_{k, k+1}\right) U_{j-k}\left(\Gamma_{k, k+2}\right) \ldots U_{j-k}\left(\Gamma_{k, j}\right)\left(U_{k+1, j} \oplus I_{\mathcal{D}_{\Gamma_{k, j}^{*}}}\right),
$$

where

$$
U_{j-k}\left(\Gamma_{k, k+l}\right)=I \oplus U\left(\Gamma_{k, k+l}\right) \oplus I .
$$

To a family of contractions, $\Gamma_{k, j}$ one can associate a row contraction via

$$
R_{k, j}=\left[\begin{array}{llll}
\Gamma_{k, k+1}, & D_{\Gamma_{k, k+1}^{*}} \Gamma_{k, k+2}, \ldots, \quad D_{\Gamma_{k, k+1}^{*}} \ldots D_{\Gamma_{k, j-1}^{*}} \Gamma_{k, j}
\end{array}\right]
$$

and a column contraction via

$$
C_{k, j}=\left[\begin{array}{llll}
\Gamma_{j-1, j}, & \Gamma_{j-2, j} D_{\Gamma_{j-1, j}}, \ldots, \quad \Gamma_{k, j} D_{\Gamma_{k+1, j}} \ldots D_{\Gamma_{j-1, j}}
\end{array}\right]^{t},
$$

where " $t$ " stands for matrix transpose. For more details on the ranges and domains of these operators see [5].

Then the main theorem regarding positive matrices can be stated informally as follows (for a precise statement, especially concerning the ranges and domains of all operators involved, see [5])

Theorem 5. The matrix $S=\left[S_{k, j}\right]_{k, j=1}^{d}$ as above, satisfying $S_{j k}^{*}=S_{k j}$, is positive if and only if i) $S_{k k} \geq 0, k=1, \ldots, d$ and ii) there exists a family $\left\{\Gamma_{k, j} \mid k, j=1, \ldots, d, k \leq j\right\}$ of contractions such that $\Gamma_{k, k}=0$ for $k=1, \ldots, d$ valid, and

$$
S_{k, j}=L_{k, k}^{*}\left(R_{k, j-1} U_{k+1, j-1} C_{k+1, j}+D_{\Gamma_{k, k+1}^{*}} \ldots D_{\Gamma_{k, j-1}^{*}} \Gamma_{k, j} D_{\Gamma_{k+1, j}} \ldots D_{\Gamma_{j-1, j}}\right) L_{j, j} .
$$

where $L_{k, k}$ is any square root of $S_{k k}$.

Definition 6. The contractions $\Gamma_{k, j}$, with $j>k$, will be called the Schur-Constantinescu parameters of $S$.

These parameters were first discovered for Toeplitz matrices by Schur, 24, albeit in the guise of a problem about power series which are bounded in the unit circle. In our humble opinion, it was our late colleague and teacher, T. Constantinescu, who championed the study of these parameters to cover all positive matrices (more generally to matrices with displacement structure, [8]) and most adroitly brought to fore many of their interesting features. Therefore, we have chosen to call these parameters, the Schur-Constantinescu parameters, in his honour.

We will illustrate Theorem (5) via the case of $3 \times 3$ positive matrices.

Thus, let $S=\left[\begin{array}{lll}S_{11} & S_{12} & S_{13} \\ S_{12}^{*} & S_{22} & S_{23} \\ S_{13}^{*} & S_{23}^{*} & S_{33}\end{array}\right]$ be a positive matrix. Then $S_{i i}>0$ and let us pick $L_{i i}$ as the positive square roots of $S_{i i}$. In this case $L_{i i}^{*}=L_{i i}$. Then per Theorem [5] there are complex numbers $\Gamma_{12}, \Gamma_{13}, \Gamma_{23}$ in the unit disc such that gives:

$$
\begin{aligned}
& S_{12}=L_{11}^{*} \Gamma_{12} L_{22}, \\
& S_{23}=\underset{22}{L_{22} \Gamma_{23} L_{33},} \\
& 6
\end{aligned}
$$




$$
S_{13}=L_{11}^{*}\left(\Gamma_{12} \Gamma_{23}+D_{\Gamma_{12}^{*}} \Gamma_{13} D_{\Gamma_{23}}\right) L_{33},
$$

Note that there is a recursive procedure to determine the $\Gamma_{k j}$. The first and the second equations yield $\Gamma_{12}, \Gamma_{23}$ from quantities already known, while the last equation yields $\Gamma_{13}$ from quantities already determined at the first two equations.

Whilst, the Schur-Constantinescu paramters are defined directly in terms of the entries of $S$, one could also seek expressions for them in terms of the vector $\beta$ of Equation (3.1) (i.e, when $S$ is a density matrix). See [7, 5] for such expressions. In particular, for $d=2$ the analogue of the Bloch sphere is now a cylinder.

It is appropriate to make several comments about these parameters at this point:

- C1 As can be expected from the form of Equation (4.2), Theorem (5) is valid for operator matrices, i.e., matrices whose entries are matrices or even operators in infinite-dimensional spaces, i.e., for elements of $\mathcal{M}_{d} \otimes \mathcal{L}(\mathcal{H})$, with $\mathcal{H}$ allowed to be infinite-dimensional. In fact, one can easily extend the result to infinite matrices with (possibly infinite-dimensional) operator entries.

- C2 Though we only called the $\Gamma_{k j}$ as the Schur-Constantinescu parameters, a full parametrization is provided by the $\frac{d(d-1)}{2}$ contractions $\Gamma_{k j}, k<j$ and the $L_{i i}, i=1, \ldots, d$. In the case of scalar valued matrices, i.e., when $\mathcal{H}=C$, we thus get the right count of $d^{2}$ real parameters. Note the $\Gamma_{k k}=0$ are just some fake parameters, included in the statement of the theorem to avoid an artificial separation of the $j=k+1$ case from that for other values of $j$.

- C3 Since the $L_{i i}$ are allowed to be any choice of square root of $S_{i i}$ (i.e., $S_{i i}=L_{i i} L_{i i}^{*}$ ), the parametrization will be different for different choices of the $L_{i i}$. A most natural choice would be the Cholesky factorization of $S_{i i}$. In fact, as described in [5], there is an algorithmic proof of Theorem (5) which automatically yields the Cholesky factorization of $S$. In the infinite-dimensional case, some of the algorithmic flavour of the proof is lost.

- C4 While, Equation (4.2) in Theorem (5) is nonlinear and looks quite complicated, there is an iterative feature to it (as mentioned in the $3 \times 3$ example given before), inasumch as in each equation there is just one of the $\Gamma_{k j}$ being solved for. It is precisely because of this that the Schur-Constantinescu parameters have an inheritance property, namely that the parameters of any leading principal submatrix (recall these will be positive themselves) are the same as that obtained from the original matrix.

- C5 Since the proof of Theorem (5) supplies the Cholesky factorization of $S$, we get an algorithmic recipe for finding one Kraus operator representation of a quantum channel $\Phi$. Since the Cholesky factor, $V$ is lower triangular, the Kraus operators, $V_{i}$, thereby obtained from $V$ (as described in Section 2), tend to be sparse. This can be useful in determining sufficient conditions for a channel to be entanglement breaking, or for computing quantities associated to channels such as the entanglement fidelity, for instance. The utility of using the Cholesky factorization lies not just in the avoidance of spectral calculations (as would be 
the case if $T$ was found from the spectral factorization of $S$ ), but that most of the Kraus operators $V_{i}$ are then sparse.

- C6 Returning to a positive matrix, $S$, whose entries are scalar, it is known that if $S_{i i}=0$, for some $i$, then the entire row and column to which $S_{i i}$ belongs has to be zero. Therefore, a reasonable convention to assume is that $\Gamma_{k j}=0$, whenever $S_{j j} S_{k k}=0$. With this convention, the $\Gamma_{k j}, L_{i i}$ provide a one-one parametrization of positive matrices.

- C7 In the previous section we saw that even the problem of characterizing pure states via the proposed parametrizations of that section was not fully resolved. However, the Schur-Constantinescu parametrization provides a very simple and effective characterization of rank one states, viz., $S$ is rank one iff all $\Gamma_{k j}=0$, except for those cases in which $S_{j j} S_{k k} \neq 0$, in which case $\Gamma_{k j}$ should be on the unit circle.

- C8 Let $S$ be positive matrix. Then there is a very simple formula for its determinant in terms of the $\Gamma_{k j}$, viz.,

$$
\operatorname{det}(S)=\left(\prod_{k=1}^{d} S_{k, k}\right) \prod_{k<j}\left(1-\left|\Gamma_{k j}\right|^{2}\right) .
$$

This is useful since some entropic quantities can often be expressed in terms of determinants, [18.

- C9 While Equation (4.2) is intricate, there is a useful diagram (called a transmission line diagram) which keeps track of all the matrix products in it.

\section{Two Further Applications}

In this section two additional applications of the parametrization of the previous section are provided. The first is to show that block Toeplitz states have positive partial transpose. The second is to examine the restrictions on the relaxation rates for an open quantum $N$-level system imposed by the requirements of complete positivity (cf., [22]).

5.1. Toeplitz States. The positive partial trace condition of [20, 12] has been found to be a very useful operational condition for entanglement. While, for general states, it is known to be necessary and sufficient only for $2 \times 2$ and $2 \times 3$ states, there have been several arguments in favour of the notion that states which satisfy this positive partial trace condition (PPT states) are "close" to being unentangled, at least inasmuch as they are not useful for tasks such as dense coding. Similarly there have been several attempts at studying the PPT property for positive matrices which satisfy additional conditions, see[2]. In this section we provide a contribution along the same vein. We show that positive Toeplitz matrices are PPT states.

The proof of this result was first found by considering the Schur-Constantinescu parameters for $3 \times 3$ block Topelitz psoitve matrices. This proof can be extended in a simple but tedious manner for $d_{i} \times d_{2}$ states. But there is, in fact, a second proof 
which works for all dimensions. We provide this first and then discuss the parameter based proof.

Proposition 7. A Toeplitz mixed state is PPT.

Let $A \in C^{N \times N}$ be a Toeplitz matrix given by

$$
\left[\begin{array}{cccc}
a_{0} & a_{-1} & \cdots & a_{-n} \\
a_{1} & a_{0} & \cdots & a_{-n+1} \\
\vdots & \vdots & \ddots & \vdots \\
a_{n} & a_{n-1} & \cdots & a_{0}
\end{array}\right]
$$

We will first, for illustration purposes, show that $A^{T}$ is also positive. This is, of course, true for arbitrary positive matrices, but it will serve to illustrate the proof in the partial transpose case. Then the $i j$-th entry of $A$ is given by $A_{i j}=a_{i-j}$. The transpose of $A$, denoted by $A^{T}$, is

$$
\left[\begin{array}{cccc}
a_{0} & a_{1} & \cdots & a_{n} \\
a_{-1} & a_{0} & \cdots & a_{n-1} \\
\vdots & \vdots & \ddots & \vdots \\
a_{-n} & a_{-n+1} & \cdots & a_{0}
\end{array}\right]
$$

, with $A_{i j}^{T}=a_{j-i}$. Next, in the cycle notation, let $\sigma_{0}$ be the element of the symmetric group $S_{N}$ on $N$ letters, $\{1,2, \cdots, N\}$, defined by

$$
\sigma_{0}=\prod_{1 \leq k \leq N}(k(N-k)) .
$$

$\sigma_{0}$ induces two simple operations on $N \times N$ matrices. If $M \in C^{N \times N}$ takes the form $M=\left[\begin{array}{c}w_{1} \\ w_{2} \\ \vdots \\ w_{n}\end{array}\right]$, where $w_{k}$ 's are rows of $\mathrm{M}$, we define the operation $R_{\sigma_{0}}$ by

$$
M \stackrel{R_{\sigma_{0}}}{\longrightarrow}\left[\begin{array}{c}
w_{\sigma_{0}(1)} \\
w_{\sigma_{0}(2)} \\
\vdots \\
w_{\sigma_{0}(n)}
\end{array}\right]
$$

, i.e. $R_{\sigma_{0}}$ simply permutes the rows of $M$ as specified by $\sigma_{0}$. Another operation on columns, $C_{\sigma_{0}}$, is define in the same way. Now we notice that if $A$ is Toeplitz as given above, then

$$
\left[R_{\sigma_{0}}\left(C_{\sigma_{0}}(A)\right)\right]_{i, j}=\underset{9}{A_{N-i, N-j}}=a_{j-i}=A_{i, j}^{T}
$$


Since $R_{\sigma_{0}}$ and $C_{\sigma_{0}}$ preserve the characteristic polynomial, we have shown that a if a Hermitian Toeplitz matrix is positive then so is its transpose.

The above fact can be extended to the partial transpose of an $N M \times N M$ Toeplitz matrix $A$ in the following way: Let $\sigma_{m}$ be the same permutation as $\sigma_{0}$ on the letters $\{m n, m n+1, \cdots,(m+1) n-1\}$. If $\sigma \in S_{N^{2}}$ is defined to be the disjoint product $\sigma_{0} \sigma_{1} \cdots \sigma_{M-1}$, and $R_{\sigma}$ and $C_{\sigma}$ are the induced operators, then by the same argument as above, we have $R_{\sigma}\left(C_{\sigma}(A)\right)=A^{P T}$, where $A^{P T}$ denotes the partial transpose of $A$. Once again these operations preserve the characteristic polynomial for Toeplitz matrices and hence if $A$ is positive, in addition, we find that so is $A^{P T}$. Thus a positive Toeplitz matrix is PPT.

The Schur parametrization of positive matrices gives another proof of proposition 1 that is immediate. If $B$ is a block Toeplitz matrix, then $B$ is also Toeplitz. So let $B$ be, for instance, a $3 \times 3$ block Toeplitz matrix. Using the Schur-Constantinescu parameters and the block Toeplitz property of $B$, we can write $B$ explicitly as

$$
\left[\begin{array}{ccc}
A & A^{\frac{1}{2}} \Gamma_{1} A^{\frac{1}{2}} & A^{\frac{1}{2}}\left(\Gamma_{1}^{2}+D_{\Gamma_{1}^{*}} \Gamma_{2} D_{\Gamma_{1}}\right) A^{\frac{1}{2}} \\
A^{\frac{1}{2}} \Gamma_{1}^{*} A^{\frac{1}{2}} & A & A^{\frac{1}{2}} \Gamma_{1} A^{\frac{1}{2}} \\
A^{\frac{1}{2}}\left(\left(\Gamma_{1}^{*}\right)^{2}+D_{\Gamma_{1}} \Gamma_{2}^{*} D_{\Gamma_{1}^{*}}\right) A^{\frac{1}{2}} & A^{\frac{1}{2}} \Gamma_{1}^{*} A^{\frac{1}{2}} & A
\end{array}\right]
$$

, where each entry is an $N \times N$ matrix. Note that due to the block-Toeplitz nature of $B$ its Schur-Constantinescu parameters $\Gamma_{i j}$ need be indexed by only one subscript. Transpose block-wise gives us $A^{P T}$. By the spectral theorem, $D_{\Gamma_{1}^{T}}=\left(D_{\Gamma_{1}}^{*}\right)^{T}$. So, simply by inspection, we see that $A^{P T}$ has Schur parameters $\left\{\left(A^{\frac{1}{2}}\right)^{T}, \Gamma_{1}^{T}, \Gamma_{2}^{T}\right\}$. Therefore $A^{P T} \geq 0$. This is in fact true in general:

Proposition 8. If $A \in C^{M N \times M N}$ is block Toeplitz, then $A$ is PPT.

The basic idea is to show that If $A$ is parametrized by $\left\{\Gamma_{i}\right\}$, then $A^{P T}$ is parametrized by $\left\{\Gamma_{i}^{T}\right\}$. Note that the block-Toeplitz property means that Schur-Constantinescu parameters of $A$ depend only on one index (cf., the $3 \times 3$ block case). We will omit the proof, which is straightforward but tedious. Via the combinatorial structure of the Schur parameters, one can see how the parametrization of $A$ gives rise to that of $A^{P T}$. The so-called "lattice structure" of the Schur parameters for the $4 \times 4$ case is shown in Figure 1 below. Each transfer box in Figure 1 describes the action of the Julia operator $U\left(\Gamma_{i}\right)$.

Let $U^{T}(\Gamma)$ denote the transpose of the Julia operator of $\Gamma$, i.e.

$$
U^{T}(\Gamma)=\left[\begin{array}{cc}
\Gamma^{T} & \left(D_{\Gamma}\right)^{T} \\
\left(D_{\Gamma^{*}}\right)^{T} & -\left(\Gamma^{*}\right)^{T}
\end{array}\right]=\left[\begin{array}{cc}
\Gamma^{T} & D_{\Gamma^{T *}} \\
\left(D_{\Gamma^{T}}\right) & -\left(\Gamma^{T}\right)^{*}
\end{array}\right]=U\left(\Gamma^{T}\right) .
$$

Each entry of the positve semidefinite kernel $\left\{A_{i j}\right\}$ corresponds to those paths in the diagram that startfrom $L_{j j}$ and end at $L_{i i}{ }^{*}$. For example, each path from $L_{33}$ to $L_{11}{ }^{*}$ describes to a summand in the expression for $A_{13}$. So we can see that the transmission 
line diagram of $A^{P T}$ is then obtained by replacing each $U\left(\Gamma_{i}\right)$ transfer box by that of $U^{T}\left(\Gamma_{i}\right)$.

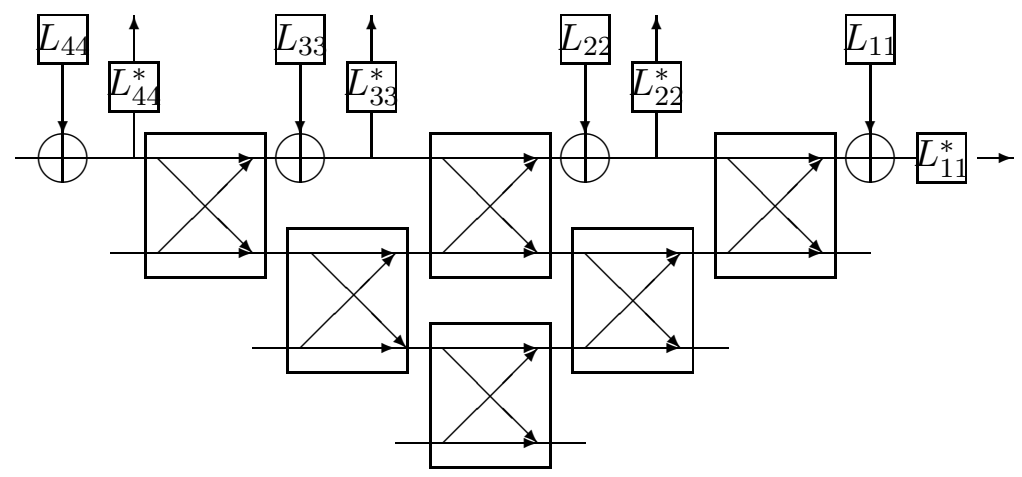

Figure 1. Lattice structure for $4 \times 4$ positive matrices

5.2. Constraints on Relaxation Rates. In this subsection we revisit the very interesting work of [22] on the constraints imposed on the relaxation rates of an open $N$-level quantum system by the requirement that its evolution be completely positive. In order to keep the notation the same as in 22, we will, in this subsection only denote the Schur-Constantinescu parameters by $g_{i j}$ (and not $\Gamma_{i j}$ ).

Let us first briefly review the contents of 22 . Let $\rho(t)$ be the state of an open $N$-level quantum system and let $\tilde{\rho}$ be the vector in $C^{N^{2}}$ which represents $\operatorname{vec}(\rho)$. Then its evolution can be expressed via

$$
\dot{\tilde{\rho}}=\left(-\frac{i}{\hbar} L_{H}+L_{D}\right) \tilde{\rho}
$$

where $L_{H}$ and $L_{D}$ are $N^{2} \times N^{2}$ matrices representing the Hamiltonian and dissipative parts respectively of the evolution of $\tilde{\rho}$. Let the index $(m, n)$ denote the number $m+(n-1) N$. Then the non-zero entries of $L_{D}$ are given by

$$
\begin{aligned}
\left(L_{D}\right)_{(m, n)(m, n)} & =-\Gamma_{m n}, m \neq n \\
\left(L_{D}\right)_{(m, m)(l, l)} & =\gamma_{m l}, m \neq l \\
\left(L_{D}\right)_{(m, m)(m, m)} & =-\sum_{k=1, k \neq m}^{N} \gamma_{k m}
\end{aligned}
$$

Here $\gamma_{k n}$ is the population relaxation rate from level $\mid n>$ to $\mid k>$. The $\gamma_{k n}$ are real and non-negative. $\Gamma_{k n}$ (for $k \neq n$ ) is the dephasing rate for the transition from $\mid k>$ to $\mid n>$. Since, $\Gamma_{k n}=\Gamma_{n k}$, it is easily seen that $\left(L_{D}\right)_{(m, n)(m, n)}=\left(L_{D}\right)_{(n, m)(n, m)}$. A key step in the work of [22] is to express $\Gamma_{k n}$ as a sum of two summands, in recognition of the fact that dephasing is also enhanced by population relaxation, to wit

$$
\Gamma_{k n}=\Gamma_{k n}^{p}+\Gamma_{k n}^{d}
$$

with $\Gamma_{k n}^{p}$, the decoherence rate due to population relaxation and $\Gamma_{k n}^{d}$ the decoherence rate due to pure phase relaxation. The requirement that the open quantum system's 
evolution be completely positive, 1, 9, imposes restrictions on $\gamma_{k n}$ and $\Gamma_{k n}$. These restrictions can be expressed as the requirement that a certain $\left(N^{2}-1\right) \times\left(N^{2}-1\right)$ matrix concocted out of the $\gamma_{k n}$ and $\Gamma_{k n}$ be positive, 22]. However, per [22], this requirement can be reduced to verifying that a related $(N-1) \times(N-1)$ matrix be positive. The form of this $(N-1) \times(N-1)$ matrix will depend on a choice of an orthogonal basis for the space of traceless, Hermitian $\left(N^{2}-1\right) \times\left(N^{2}-1\right)$ matrices. However, positivity of this matrix itself is independent of the choice of basis. The excellent analysis of [22] is unfortunately marred for the $N=4$ case by an incorrect criterion for positivity. Indeed, Equation (28) of [22 are only necessary for positivity, while Equations (31)-(32) are (as correctly claimed in [22]) also just necessary (though they come closer to sufficiency than Equation (28) of [22]).

In the sequel, we will use the Schur-Constantinescu parameters to analyse the $N=4$ case of [22. As in [22] the evolution Equation (5.1) is completely positive iff the $3 \times 3$ real symmetric matrix $B=\left(b_{i j}\right)$ is positive. To specify the entries of the $b_{i j}$, we denote by $\Gamma_{\text {tot }}^{d}$ the quantity $\frac{1}{2} \sum_{n=2}^{4} \sum_{m=1}^{n-1} \Gamma_{m n}^{d}$. Then the entries of $B$ are given by

$$
\begin{aligned}
& b_{11}=\Gamma_{t o t}^{d}-\left(\Gamma_{13}^{d}+\Gamma_{24}^{d}\right. \\
& b_{22}=\Gamma_{t o t}^{d}-\left(\Gamma_{13}^{d}+\Gamma_{24}^{d}\right. \\
& b_{33}=\Gamma_{t o t}^{d}-\left(\Gamma_{12}^{d}+\Gamma_{34}^{d}\right. \\
& b_{12}=\frac{\left(\Gamma_{12}^{d}-\Gamma_{34}^{d}\right)}{2} \\
& b_{13}=\frac{\left(\Gamma_{14}^{d}-\Gamma_{23}^{d}\right)}{2} \\
& b_{23}=\frac{\left(\Gamma_{13}^{d}-\Gamma_{24}^{d}\right)}{2}
\end{aligned}
$$

Now $B$ is positive iff $b_{i i} \geq 0, i=1, \ldots, 3$ and the Schur-Constantinescu parameters $g_{12}, g_{13}, g_{23}$ are in the closed unit disc. Since $B$ is real this is equivalent to demanding that the $g_{i j}$ belong to the interval $[-1,1]$.

The conditions $b_{i i} \geq 0$ become

$$
\begin{aligned}
& \Gamma_{12}^{d}+\Gamma_{14}^{d}+\Gamma_{23}^{d}+\Gamma_{34}^{d} \geq \Gamma_{13}^{d}+\Gamma_{24}^{d} \\
& \Gamma_{12}^{d}+\Gamma_{13}^{d}+\Gamma_{24}^{d}+\Gamma_{34}^{d} \geq \Gamma_{14}^{d}+\Gamma_{23}^{d} \\
& \Gamma_{13}^{d}+\Gamma_{14}^{d}+\Gamma_{23}^{d}+\Gamma_{24}^{d} \geq \Gamma_{12}^{d}+\Gamma_{34}^{d}
\end{aligned}
$$

Now $b_{12}=\sqrt{b_{11}} g_{12} \sqrt{b_{22}}$. So $g_{12} \in[-1,1]$ becomes

$4 \Gamma_{12}^{d} \Gamma_{34}^{d}-\left(\Gamma_{13}^{d}-\Gamma_{14}^{d}\right)^{2}-\left(\Gamma_{13}^{d}-\Gamma_{23}^{d}\right)^{2}+\left(\Gamma_{13}^{d}-\Gamma_{24}^{d}\right)^{2}+\left(\Gamma_{14}^{d}-\Gamma_{23}^{d}\right)^{2}-\left(\Gamma_{14}^{d}-\Gamma_{24}^{d}\right)^{2}-\left(\Gamma_{23}^{d}-\Gamma_{24}^{d}\right)^{2} \geq 0$

Likewise the condition $g_{23} \in[-1,1]$ becomes

$4 \Gamma_{13}^{d} \Gamma_{24}^{d}-\left(\Gamma_{12}^{d}-\Gamma_{14}^{d}\right)^{2}-\left(\Gamma_{12}^{d}-\Gamma_{23}^{d}\right)^{2}+\left(\Gamma_{12}^{d}-\Gamma_{34}^{d}\right)^{2}+\left(\Gamma_{14}^{d}-\Gamma_{23}^{d}\right)^{2}-\left(\Gamma_{14}^{d}-\Gamma_{34}^{d}\right)^{2}-\left(\Gamma_{23}^{d}-\Gamma_{34}^{d}\right)^{2} \geq 0$

Finally $g_{13} \in[-1,1]$ becomes

$$
b_{11} b_{22} b_{33}+2 b_{12} b_{13} b_{23} \geq b_{12} b_{23}^{2}+b_{22} b_{13}^{2}+b_{33} b_{12}^{2}
$$


Note that the condition $\left|g_{13}\right| \leq 1$ is not similar to the condition for the other $g_{i j}$ to be in $[-1,1]$. This is to be expected since the formula for $g_{j k}$ for $k>j+1$ is more intricate than those for the $g_{j k}, k=j+1$. Furthermore, this last condition is precisely one of those obtained in 22]. However, the conditions obtained here are necessary and sufficient.

\section{Conclusions}

Since positive matrices play a vital role in many applications, it is of importance to obtain computable parametrizations of them. In this paper we discussed several such potential parametrizations. Which one of them one ought to use is, of course, a matter dictated by the application one has in mind. We argued, hopefully persuasively, in favour of the versatility of the parametrization proposed in [5]. There are several other applications besides the ones discussed here, to which one could apply this parametrization. This will be the subject of future work.

\section{REFERENCES}

[1] R.Alicki, K. Lendi, Quantum Dynamical Semigroups and Applications, Springer-Verlag, Berlin (1987).

[2] S. Braunstein, S. Ghosh, S. Severini, The laplacian of a graph as a density matrix, arXiv, quant-ph 0406165, (2004).

[3] M. Byrd, N. Khaneja, Chaarcterization of the positivity of the density matrix in terms of the coherence vector representation, Phys. Rev A, 68, 062322 (2003).

[4] M. D. Choi, Completely positive linear maps on complex matrices, Lin. Alg. Appl., 10(1975), $285-290$.

[5] T. Constantinescu, V. Ramakrishna, Parametrizing quantum states and channels, Quantum Information Processing, 2, 221-248, 2003.

[6] T. Constantinescu, Schur Parameters, Factorization and Dilation Problems, Birkhäuser, 1996.

[7] T. Constantinescu, V. Ramakrishna, On a parametrization of purifications of a qubit, Quantum Information Processing, 1, No 5, 109-124, 2003.

[8] T. Constantinescu, A. H. Sayed, T. Kailath, Displacement structure and completion problems, SIAM J. Matrix Anal. Appl., 16(1995), 58-78.

[9] V. Gorini, A. Kossakowski, E. Sudarshan, J. Math Physics, 17, 821 (1976).

[10] T. F Havel, Procedures for converting among Lindblad, Kraus and matrix representations of quantum dynamical semigroups, J. Math Physics, 44(2003), 534-557.

[11] R. Horn, C. Johnson, Matrix Analysis, Cambridge University Press, Cambridge (1986).

[12] M. Horodecki, P. Horodecki and R. Horodecki, Separability of mixed states: necessary and sufficient conditions, Phys. Lett, A223 (1996), 1.

[13] A. Jamiolkowski, Linear transformations which preserve and positive semidefiniteness of operatores, Rep Math Phys., 3 (1972), 275-278.

[14] T. Kailath, A. H. Sayed, Displacement structure: theory and applications, SIAM Rev., 37(1995), $297-386$.

[15] G. Kimura, The Bloch vector for $N$-level systems, arXiv:quant-ph/0301152 2003.

[16] K. Kraus, General state changes in quantum theory, Ann. Physics, 64(1971), 311-335.

[17] M. Nielsen, I. Chuang, Quantum Computation and Quantum Information, Cambridge University Press, 1999.

[18] M. Ohya, D. Petz, Quantum entropy and its use, Springer, Berlin, 1993. 
[19] V. Paulsen, Completely bounded maps and dilation, Pitman Research Notes in Math. 146, Longman, Wiley, New York, 1986.

[20] A. Peres, Separability criterion for density matrices, Phys. Review Lett., 77(1996), 1413-1415.

[21] J. Preskill, website for Physics 219, www.theory.caltech.edu/people/preskill/ph229/

[22] S. G. Schirmer, A. Solomon, Constraints on the relaxation rates for $N$-level systems, Phys. Rev A, 70, 022107 (2004).

[23] S. G. Schirmer, T. Zhang, J. Leahy, Orbits of quantum states and geometry of Bloch vectors for $N$-Level systems, J. Phys A, 37 (4), 1389-1402, (2004).

[24] I. Schur, Über potenzreihen die im Inneren des Einheitskreises beschränkt sind, J. Reine und Angewandte Mathematik, 147, 205-232 (1917) (english translation in Operator Theory: Advances and Applications, 18, 31-88, I. Gohberg ed, Birkhauser, Boston, 1986).

[25] W. F. Stinespring, Positive functions on $C^{*}$ algebras, Proc. Amer. Math. Soc., 6(1955), 211-216.

[26] F. Verstraete, H. Verschelde, On quantum channels, arXiv, quant-ph 0202124 (2002).

[27] P. Zanardi, A note on quantum cloning in $d$ dimensions, arXiv:quantum-ph/9804011 v3, 1998.

Department of Mathematics, University of Texas at Dallas, Box 830688, RichardSON, TX 75083-0688, U. S. A.

E-mail address: vish@utdallas.edu 